\title{
Radioprotection et interventions de radiographie industrielle*
}

\author{
G. ABELA ${ }^{1}$
}

(Manuscrit reçu le 12 mai 2005, accepté le 16 novembre 2005)

RÉSUMÉ La division production nucléaire (DPN) d'EDF est une grande utilisatrice de contrôles radiographiques industriels : de l'ordre de 40000 contrôles sont réalisés annuellement sur le parc nucléaire pour la réalisation d'examens non-destructifs (END). Les sources ponctuelles d'iridium-192 sont le plus souvent utilisées. L'activité de ces sources peut aller, dans des configurations usuelles, jusqu'à 4,4 TBq $(120 \mathrm{Ci})$, induisant un débit d'équivalent de dose de l'ordre de $0,54 \mathrm{~Sv} \mathrm{~h}^{-1}$ à 1 mètre. À cette distance, la valeur de $20 \mathrm{mSv}$ (limite annuelle pour les travailleurs) est atteinte en un peu plus de 2 minutes. Ce jour, aucun dépassement des limites réglementaires n'a été observé à la DPN EDF, mais dans le cadre de l'observation des évènements, pratiquée depuis de nombreuses années, des «signaux faibles", ainsi que des expositions non prévues inférieures aux limites réglementaires ont été notés. La direction de la DPN a donc décidé de mener des actions particulières dans le domaine.

ABSTRACT Radiation protection and inspection of industrial radiography.

The EDF nuclear power division (NPD) conducts a substantial number of industrial $\gamma$-ray inspections: about 40.000 inspections are conducted annually on the nuclear fleet. Point sources of iridium-192 are generally used. In normal configurations, the activity of these sources can go up to $4.4 \mathrm{TBq}(120 \mathrm{Ci})$, inducing - at a range of 1 meter - such a high dose equivalent rate that the value of $20 \mathrm{mSv}$ is reached in a little more than 2 minutes. To date, no exceeding of the regulatory limit has been observed within EDF, but during the observation of events, a practice that has been in effect for many years, "low-level events", such as unplanned exposures lower than the regulatory limits, were noted. NPD management thus decided to take particular actions in the field. An analysis of unplanned exposures was carried out.

Keywords: Radiation protection / Gamma-ray inspection / ergonomics

L'activité peut paraître « simple » car elle est composée de taches élémentaires qui paraissent, prises isolément, facilement réalisables. En fait, la réalisation de ces taches :

- fait appel à des connaissances techniques très importantes ;

\footnotetext{
* L'analyse «facteur humain » a bénéficié de l'aide d'une équipe d'ergonomes C. Tannière, G. Carballeda, et A. Garrigou, de l'université de Bordeaux.

1 EDF, Centre d'appui du parc en exploitation, Groupe prévention des risques environnement, 1 place Pleyel, 93282 SaintDenis Cedex 2, France.
} 
- demande un dossier de préparation complexe à rédiger car il nécessite de nombreuses interfaces avec d'autres interlocuteurs ;

- est en lien avec d'autres activités réalisées obligatoirement en amont du contrôle radiographique ;

- nécessite une préparation des zones à baliser pour en interdire l'accès ;

- induit l'adaptation à des conditions physiques (température, éclairage, bruit, débit de dose) et d'accessibilité parfois très contraignantes.

«L'erreur» de l'opérateur, son manque d'attention ou de vigilance ne peuvent donc pas être considérés par un préventeur comme des cause d'incident ou d'accident satisfaisante dans le cadre d'une démarche de progrès.

L'analyse des causes matérielles n'a pas mis en évidence de cause liée au matériel utilisé (le «gammagraphe »). Il s'agit de matériel très fiable, robuste, mais dont l'ergonomie est perfectible.

L'évolution des prescriptions a porté sur:

- le port obligatoire et systématique de dosimètres opérationnels munis d'alarme sonore ;

- l'optimisation des limites de débit d'équivalent de dose (DED) acceptables en limite de balisage ;

- une analyse de risque spécifique a toujours été menée sur les sites EDF, son résultat est traduit par le « permis de contrôle radiographique »;

- la gestion des sources radio actives : le référentiel radioprotection du parc en exploitation prévoit que toutes les sources présentes sur les sites soient soumises aux mêmes règles de gestion, qu'elle appartiennent à EDF ;

- l'utilisation de nouveau matériel de signalisation et de mesure.

En conclusion, les pistes actuelles qui doivent permettre d'assurer une meilleure qualité et une meilleure sécurité dans la mise en œuvre nous semblent être :

- une meilleure planification de ces activités de contrôle au sein des projets ;

- l'augmentation du contrôle des activités ;

- le partage d'informations de l'ensemble du personnel présent sur l'installation ;

- amélioration et la formalisation du dialogue entre l'entreprise utilisatrice et l'entreprise intervenante ;

- la formation des opérateurs.

\section{Introduction}

La division production nucléaire (DPN) d'EDF est une grande utilisatrice de contrôles radiographiques industriels : de l'ordre de 40000 contrôles sont réalisés 
annuellement sur le parc nucléaire (58 tranches) pour la réalisation d'examens non destructifs (END). Ces examens sont réalisés dans le cadre d'un programme de base de maintenance préventive. Des contrôles sont aussi réalisés dans le cadre de réparation de matériels, tels que des changements de robinets par exemple, il s'agit de « contrôles non destructifs » (CND).

Les sources ponctuelles d'iridium-192 sont le plus souvent utilisées. L'activité de ces sources peut aller, dans des configurations usuelles, jusqu'à 4,4 TBq $(120 \mathrm{Ci})$, induisant un débit d'équivalent de dose de l'ordre de $0,54 \mathrm{~Sv} \mathrm{~h}^{-1}$ à 1 mètre. À cette distance, la valeur de $20 \mathrm{mSv}$ (limite annuelle pour les travailleurs) est atteinte en un peu plus de 2 minutes.

Des accidents d'exposition survenus dans le cadre de la radiographie industrielle sont décrits par l'AIEA (voir http://www.iaea.org). Ces accidents ont parfois des conséquences très graves et peuvent toucher le public ou les intervenants manipulant ou travaillant à proximité. Ne seront abordés ici que les risques concernant des travailleurs intervenants dans des installations industrielles.

Cette présentation expose le point de vue d'un «client», tel que décrit par l'AIEA (1999). Ce client est particulier, du fait de sa qualité d'exploitant nucléaire, mais les risques sont les mêmes que ceux de tout autre industriel.

Une classification récente des évènements significatifs en radioprotection (ESR) nous conduit à déclarer des évènements. Ceux-ci sont classés selon un des critère suivant, selon les circonstances entre le $1^{\text {er }}$ janvier 2004 et le 15 novembre 2005 :

- ESR critère 2 : «... situation imprévue ayant entraîné un dépassement du quart d'une limite de dose individuelle annuelle » : (1 événement, en juin 2004) ;

- ESR critère 4: «Toute activité notable comportant un risque radiologique, réalisée sans une analyse de radioprotection, ... ou sans prise en compte exhaustive de cette analyse ... » (2 évènements : intervenants présents dans la zone d'exclusion);

- ESR critère 6: «Tout défaut de signalisation de zone contrôlée » (10 évènements : défauts de balisage) ;

- ESR critère 10 : «tout autre écart significatif » (18 évènements déclarés sur décision managériale : défauts divers).

À ce jour, aucun dépassement des limites réglementaires n'a été observé à EDF DPN, mais dans le cadre de l'analyse des évènements, pratiquée depuis de nombreuses années, des « signaux faibles », ainsi que des expositions non prévues inférieures aux limites réglementaires ont été notés. La direction de la DPN a donc décidé de mener des actions particulières dans le domaine. 


\section{L'analyse des évènements}

Tout d'abord, une analyse des expositions non prévues a été menée, en concertation entre les sites concernés, le centre d'expertise et d'inspection dans les domaines de la réalisation et de l'exploitation (CEIDRE) et le groupe prévention des risques (GPR).

Les conclusions de pratiquement tous les évènements mettent en évidence «l'erreur de l'intervenant » : «l'intervenant a oublié de... », « l'intervenant n'a pas vérifié... » etc. L'expérience importante de EDF dans le domaine de l'analyse des événements relatifs à la sûreté des installations nous a conduit à réaliser une analyse multi-causes des évènements. Cette analyse a porté principalement sur :

- le «facteur humain »;

- les causes matérielles.

\subsection{L'analyse «facteur humain »}

Elle a bénéficié de l'aide d'une équipe d'ergonomes C. Tannière, G. Carballeda, sous la direction de A. Garrigou, de l'université de Bordeaux (Garrigou et al., 2003).

Cette étude (EAN, 2002) a montré que le contrôle radiographique est une activité nocturne, à risque, peu connue réalisée à l'aide de matériels fiables.

Activité nocturne car sur les sites EDF, les contrôles sont réalisés au cours des arrêts pour rechargement du combustible, à des moments de plus faible occupation des locaux. Ceci a pour but de limiter le risque d'exposition de personnel, mais aussi de limiter l'impact sur le planning de la condamnation des locaux. Cette pratique est conforme à celle utilisée par l'industrie.

Activité à risque du fait de l'activité des sources, de l'étendue des zones d'interdiction, et du nombre d'interfaces avec d'autres activités.

Activité peu connue car, réalisée de nuit, très peu d'acteurs de l'exploitation des installations sont présents. L'activité n'est connue que par ses résultats, tels qu'une non conformité de soudure, ..., rarement par ses impératifs de mise en œuvre.

Pour les observateurs, cette activité est «simple» (voir Fig. 1). En fait, la réalisation de ces tâches :

- fait appel à des connaissances techniques très importantes, mais aussi des connaissances sur l'organisation du site où se déroule le contrôle ; 


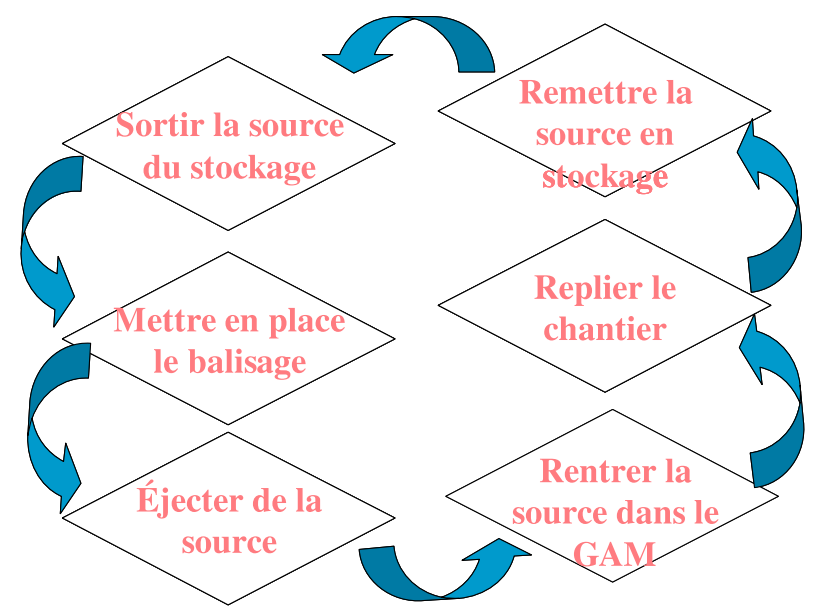

Figure 1 - L'enchaînement des actions simples.

The sequence of the simple actions.

- demande un dossier de préparation complexe à rédiger car il nécessite de nombreuses interfaces avec d'autres interlocuteurs que ceux de l'entreprise intervenante ;

- est en lien avec d'autres activités réalisées obligatoirement en amont du contrôle radiographique. Ces activités sont par exemple les activités de décalorifugeage, de «nettoyage » (ou brossage) des soudures à contrôler, de retrait des protections biologiques posées sur les circuits, ... ;

- nécessite une préparation des zones à baliser pour en interdire l'accès ;

- induit l'adaptation à des conditions physiques (température, éclairage, bruit, débit de dose) et d'accessibilité parfois très contraignantes.

«L'erreur » de l'opérateur, son manque d'attention ou de vigilance, ne peuvent donc pas être considérés par un préventeur comme des causes d'incident ou d'accident objectives dans le cadre d'une démarche de progrès.

Les conclusions de l'étude menée par A. Garrigou et son équipe portent sur la multi-causalité des accidents, la perte de vigilance étant induite par de nombreux facteurs qui sont résumés par la figure 2 : la vigilance, c'est du travail.

\subsubsection{Organisation dans l'action}

Il est nécessaire de gérer les intrusions éventuelles dans le balisage par du personnel n'ayant pas la connaissance du niveau de risque induit par l'utilisation de sources radioactives. 


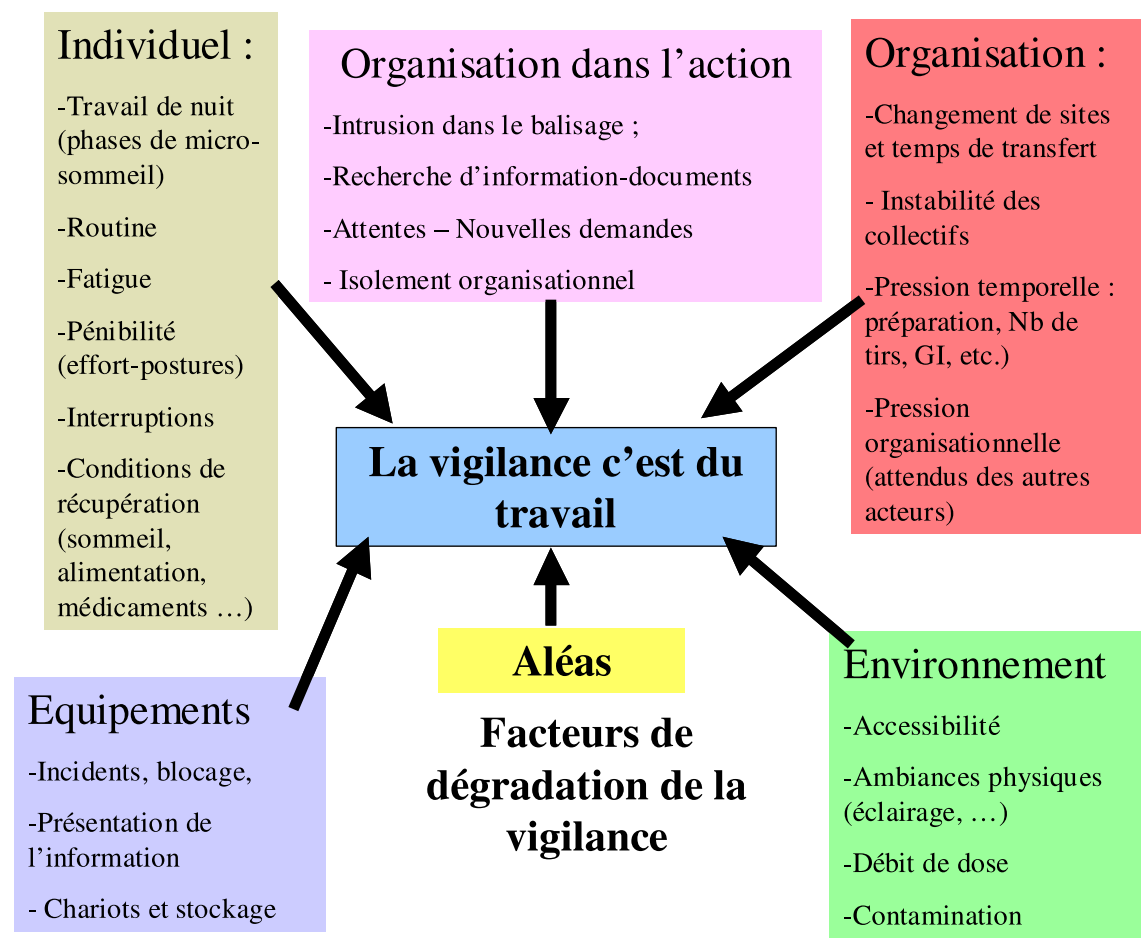

Figure 2 - Facteurs de dégradation de la vigilance.

Factors of degradation of vigilance.

Le travail nocturne induit un isolement organisationnel, puisque le personnel donneur d'ordre de l'intervention est absent, toute demande d'information complémentaire est problématique, et tout aléa, même mineur, induit des temps d'attente importants.

\subsubsection{La charge individuelle}

Le travail de nuit induit toujours des phases de micro sommeil, particulièrement pour ce personnel qui est «en déplacement », les conditions de récupération ne sont donc pas toujours optimum.

\subsubsection{Les équipements}

Le matériel (Fig. 3) est fiable, puisque aucun événement n'a été observé du fait d'une panne. Mais ce matériel est lourd : de l'ordre de $12 \mathrm{~kg}$ pour le gammagraphe, 


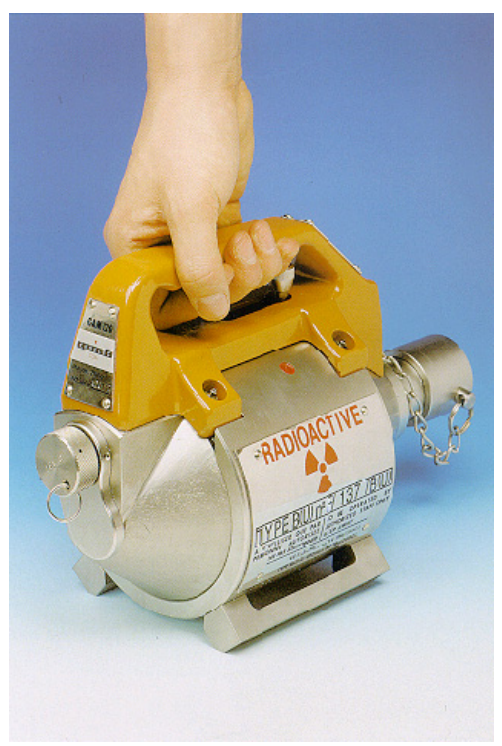

Figure 3-Le Gammagraphe en configuration de transport (image fournie par CEGELEC). Camera in shipping configuration.

poids auquel il faut ajouter les cassettes de film qui comportent des écrans de plomb et tout le matériel de balisage.

Cependant, lors de la manipulation, l'information sur la position de la source (Figs. 4-7) n'est pas facilement visible, notamment dans des conditions d'éclairage dégradées.

La manivelle (Figs. 8 et 9) de commande doit être manipulée avec la plus grande « délicatesse » et la plus grande attention.

\subsubsection{L'environnement}

Le travail se fait dans un environnement industriel où sont présentes les contraintes habituelles liées au bruit, à un éclairage pas toujours adapté à des travaux de précision (Fig. 10), à l'accessibilité difficile (Fig. 11), mais qui impose aussi les contraintes liées à le zone contrôlée. La présence de débits d'équivalent de dose et parfois de contamination peuvent nécessiter la mise en place de protections collectives telles que des protections biologiques, mais aussi de protections individuelles comme des tenues complémentaires, ventilées ou non, selon le niveau de risque. 


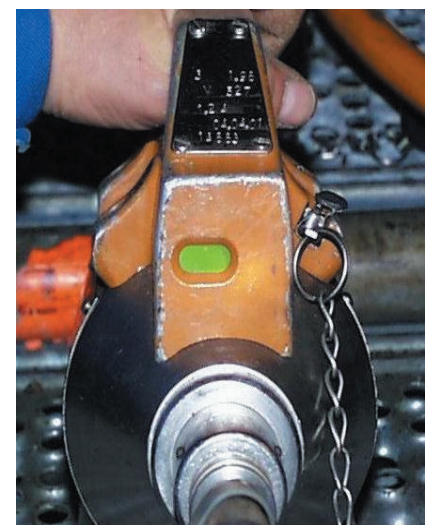

Figure 4-Signal vert: le projecteur est en position de stockage, la source rentrée et l'appareil verrouillé.

Green signal: the camera is in position of storage, and locked.

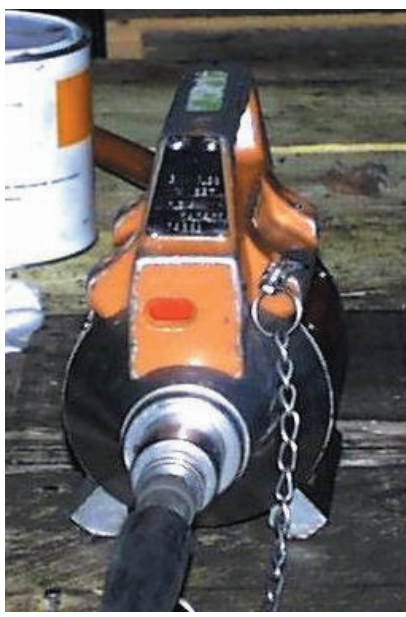

Figure 6- Signal rouge : l'exposition peut être réalisée, l'obturateur est ouvert après action sur le loquet d'armement prévu à cet effet, la source est éjectée ou prête à être éjectée.

Red signal: the exposure can be carried out, the obturator is open after action on the latch of armament envisaged for this purpose, the source is ejected or lends to being ejected.

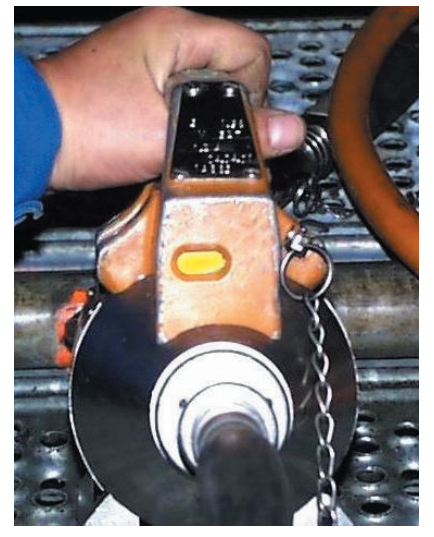

Figure 5 - Signal jaune : le projecteur est sur le chantier, la source rentrée et l'appareil déverrouillé par sa clé de sécurité individuelle.

Yellow signal: the camera in on work place, source inside, and un-locked.

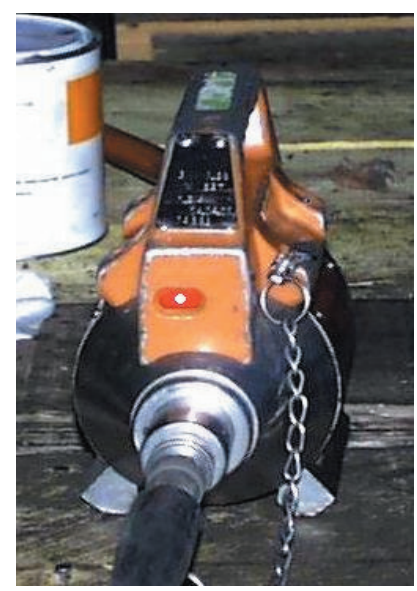

Figure 7 - Signal rouge, point blanc: en phase d'incident, la rentrée du portesource est incomplète.

Signal red, not white: in phase of incident, the re-entry of the carrysource is incomplete. 
RADIOPROTECTION ET INTERVENTIONS DE RADIOGRAPHIE INDUSTRIELLE

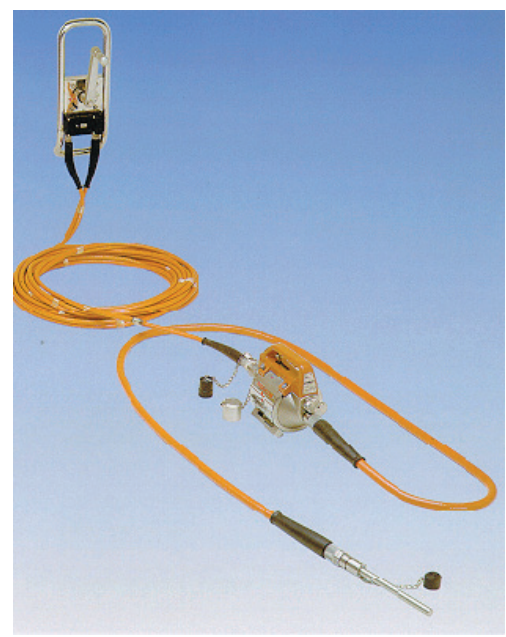

Figure 8 - Le gammagraphe en configuration d'utilisation (image fournie par CEGELEC).

Camera in configuration of use.

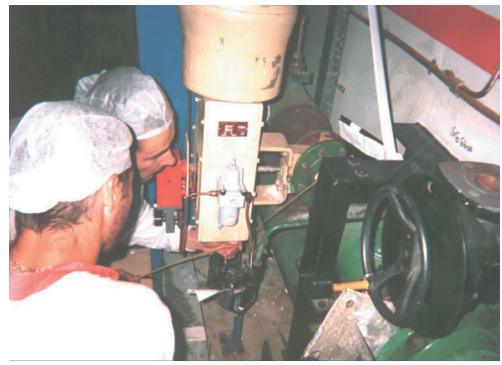

Figure 10 - Prise de cotes pour radiographier. Catch of dimensions to radiograph.

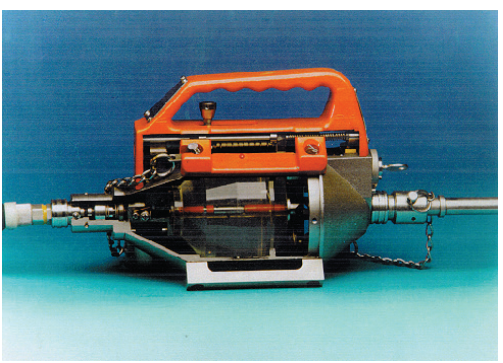

Photo 9-Le gammagraphe en coupe (image fournie par CEGELEC)

Camera section.

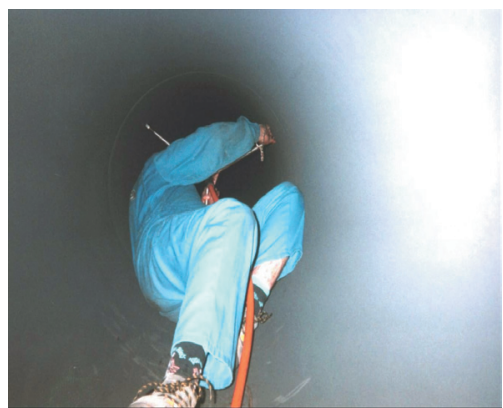

Figure 11 - Mise en place du porte source dans une tuyauterie.

Installation of the source holder in a pipe. 


\subsubsection{L'organisation}

Le personnel en charge des contrôles radiographique est hautement spécialisé, il ne fait donc pas partie des effectifs habituellement présents pour l'exploitation du site. Ce personnel fait partie d'entreprises titulaires de contrats nationaux, gérés par les services centraux de EDF (pour les END) et sont amenés à se déplacer régulièrement de site en site. Ces déplacements induisent des temps de transfert qui sont parfois longs, et une restructuration quasiment systématique des équipes. Cette «instabilité des collectifs » nécessite une réorganisation permanente des équipes qui travaillent toujours en binômes.

La pression temporelle est aussi très importante, du fait du coût d'immobilisation des installations, mais aussi par les conséquences du résultat des contrôles sur l'activité des autres métiers tels que les mécaniciens, les soudeurs, etc.

\subsection{L'analyse des causes matérielles}

Elle n'a pas mis en évidence de cause liée au matériel utilisé (le « gammagraphe »). Il s'agit de matériel très fiable, robuste, mais dont l'ergonomie est perfectible. Son statut de conteneur de transport de type B empêche toute évolution, même mineure qui demanderait une requalification économiquement non envisageable du fait du volume très faible du marché. Si on compare le matériel utilisé en France avec d'autres projecteurs utilisés aux Etats Unis ou dans d'autres pays européen, le projecteur français, constitue la référence. Si on ne peut pas écarter l'amélioration du matériel, cette piste n'est pas la première à devoir être mise en œuvre, car coûteuse, et offrant peu de garantie de résultat. L'automatisation des projecteurs, à l'aide de commandes électriques et d'automatisme associés est à proscrire totalement dans le cas de contrôles sur chantier. Le poids, l'encombrement et le temps de mise en œuvre sont des facteurs qui emmènent à écarter cette hypothèse.

Afin de prendre au mieux en compte les conclusions de l'étude ergonomique, EDF DPN avec l'appui du CEIDRE a fait évoluer ses prescriptions internes et a proposé aux sites utilisateurs de contrôles radiographiques des «bonnes pratiques ». Ces pratiques sont, bien sûr mises en œuvre en complément des mesures réglementaires.

\subsection{La réglementation (liste non exhaustive)}

- L'arrêté du 25 juin 1987 a été repris dans son principe par l'article R 231-91 du code du travail (décret du 31 mars 2003) impose que : tout intervenant 
manipulant un gammagraphe, ou un générateur de rayons $\mathrm{X}$ à usage industriel doit être titulaire du certificat d'aptitude à manipuler les appareils de radioscopie industrielle et de radiographie industrielle (le CAMARI). Ce certificat est valable pendant 9 ans si l'intervenant peut justifier d'une pratique depuis moins de trois ans.

- L'arrêté du 2 mars 2004 : «Conditions particulières d'emploi applicable aux dispositifs destinés à la radiographie industrielle utilisant le rayonnement gamma » précise les conditions de mise en œuvre, et impose notamment que pour les contrôles radiographiques réalisés sur site, l'équipe soit constituée obligatoirement de deux personnes.

- Les appareils et leurs accessoires (télécommande et gaine d'éjection) doivent être révisés tous les ans. Un carnet de suivi accompagne l'appareil.

\section{Les prescriptions internes EDF}

\subsection{Stockage des sources}

Les prescriptions imposent le stockage des sources sur le site est réalisé sous la responsabilité d'EDF : à leur arrivée sur le site, la source est remise à EDF qui assure son stockage et qui vérifie notamment avant chaque utilisation qu'un permis de contrôle radiographique a été délivré.

\subsection{Permis de contrôle radiologique}

Les prescriptions imposent la rédaction d'un « permis de contrôle radiologique » (Fig. 12).

Ce « permis de contrôle radiologique » a évolué sur les différents sites, un modèle unique sur le "parc » est prescrit pour homogénéiser les pratiques. Ce document peut être assimilé à un complément réactif au plan de prévention visant à partager les informations, et à coordonner les parades.

\subsection{Dosimètre à alarme sonore}

Les prescriptions imposent le port de dosimètres à alarme sonore, quel que soit le lieu de contrôle radiographie : en zone contrôlée ou hors zone contrôlée. L'analyse des évènements en France et à l'étranger montre que les accidents d'irradiations font suite à une succession d'erreurs, dont le retour de la source dans le projecteur qui n'a pas été réalisé, et la mesure de débit d'équivalent de dose (DeD) qui n'est pas faite. L'analyse facteur humain a confirmé qu'il n'est pas possible d'obtenir 


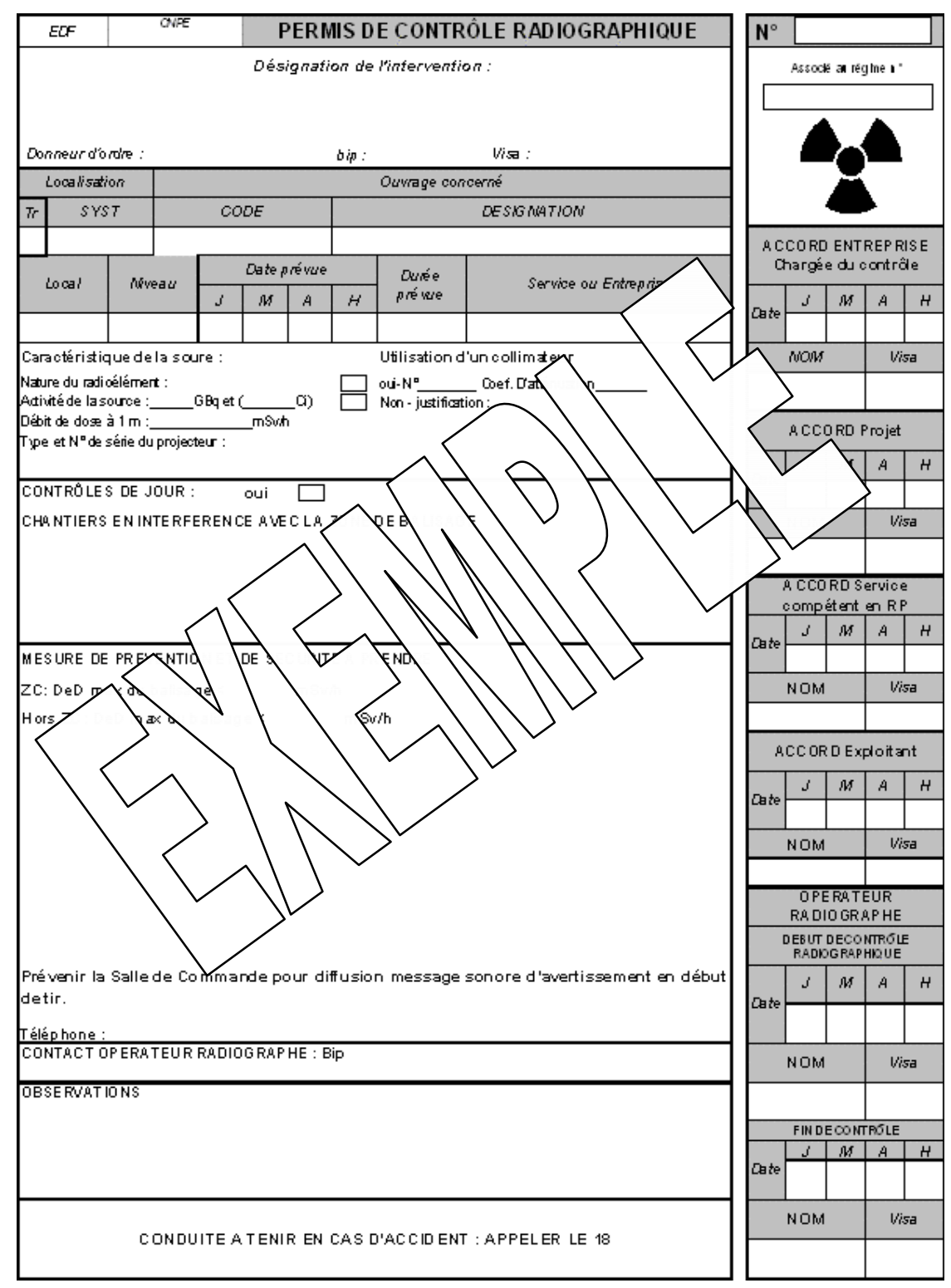

Figure 12 - Exemple de permis de contrôle radiographique.

Example of radiological licence of control. 
des intervenants une assurance absolue du respect de ces règles, il a donc été décidé de rendre obligatoire un système passif. Ces dosimètres électroniques disposent d'alarmes sonores réglées à un seuil relativement haut pour ne pas pouvoir être confondu avec une évolution du DeD, usuelles sur site nucléaire.

\subsection{Débit d'équivalent de dose}

Elles imposent la valeur de débit d'équivalent de dose en limite de balisage, est définie en fonction de la durée des tirs, de la direction de l'exposition, afin de ne pas dépasser les limites acceptables pour le public lorsque le tir a lieu hors zone contrôlée, ou pour les travailleurs de catégorie A lorsque le tir a lieu en zone contrôlée. La valeur de $7,5 \mu \mathrm{Sv} \mathrm{h}^{-1}$ hors zone contrôlée peut être utilisée comme référence en l'absence d'analyse particulière. En effet, il a été observé que l'étendue des zones interdites est un obstacle majeur à leur étanchéité. La réglementation actuelle limitant à $1 \mathrm{mSv} / \mathrm{an}$ l'exposition du public, il doit être tenu compte de la durée maximum possible de l'exposition. Les simulations suivantes ont été faites :

(i) hypothèses :

- une personne du public travaille toute l'année sur le site valeur majorant : 200 jours,

- on estime que l'on peut réaliser 20 tirs par jour dans la salle des machines qui est hors zone contrôlée, donc avec du personnel membre du «public ». Cette valeur est très supérieure à tout le retour d'expérience fait, notamment du fait que les contrôles ne sont réalisés que pendant les périodes d'arrêt pour rechargement (voir ci-après),

- chaque tir dure $2 \mathrm{~min}$,

- la personne du public se trouve en limite de balisage pendant tous les tirs,

- le balisage est fait à l'isodose $7,5 \mu \mathrm{Sv} \mathrm{h}^{-1}$,

- calcul de la dose : $200 \times 20 \times 2 / 60 \times 7,5=1000 \mu \mathrm{Sv}$,

- conclusion : dans cette configuration il est évident qu'une personne du public qui se trouve sur le site ne pourra en pas intégrer plus de $1 \mathrm{mSv}$ par an dû au tirs radio ;

(ii) estimation plus réaliste, détermination d'une limite acceptable

En réalité on ne tire jamais 200 jours dans l'année. On estime que l'on peut tirer 30 jours par an (lié à la durée des arrêts pour rechargement). Au maximum on réalisera 15 tirs par jour en salle des machines. Donc, $15 \times 30=450$ tirs par an (de l'ordre de $\mathbf{5 0 0}$ tirs par an). 
Pour respecter les $1 \mathrm{mSv}$ annuel pour une personne du public qui se trouverait en limite de balisage pendant tous les tirs de l'année, le balisage serait mis en place à l'isodose :

$$
1 /(500 \text { tirs/an } \times 2 / 60 \text { heures }) \times 1000 \mu \mathrm{Sv}=60 \mu \mathrm{Sv} \mathrm{h}^{-1} \text {. }
$$

On voit ici que les valeurs de DED utilisées pour la limite de balisage doivent absolument rester « raisonnables », et que la valeur de 7,5 $\mu \mathrm{Sv} \mathrm{h} \mathrm{h}^{-1}$ est acceptable.

Pour information, en utilisant une source de 4,44 TBq $(120 \mathrm{Ci})$ de ${ }^{192} \mathrm{Ir}$, l'isodose $0,5 \mu \mathrm{Sv} \mathrm{h}^{-1}$ se situe entre 300 et $400 \mathrm{~m}$. L'utilisation d'une telle valeur induirait tant de problème d'accessibilité des installations qu'elle est impossible à mettre en œuvre. La probabilité de non exhaustivité des balisages est aussi trop importante, le risque de pénétration généré est largement supérieur au gain espéré.

\subsection{Affichage}

Elles imposent la création d'un espace d'affichage exclusivement consacré aux permis de contrôle radio.

\subsection{Plan de balisage}

Elles imposent l'utilisation de plans type pour élaborer le plan de balisage. Ces plans, sur la base de plans des locaux validés par chaque site, sont archivés pour chaque contrôle radiographique dans un même ensemble de locaux. Ils sont accompagnés d'une feuille de calcul EXCEL indiquant les valeurs de protection propres à la source radioactive utilisée. Ces plans permettent d'optimiser les moyens mis en œuvre pour réaliser les analyses de risques en utilisant le retour d'expérience des interventions identiques.

\subsection{Balisages}

Elles imposent l'amélioration des balisages :

- des balises mises en place à proximité du gammagraphe déclenchent dés la sortie de la source (Fig. 13) et s'éteignent à son retour dans le projecteur, en remplacement des gyrophares actuellement utilisées (Sté CARMELEC) ;

- des balises mises en place en limite de balisage émettant un signal plus visible (Fig. 14) que les balises lumineuses actuellement utilisées, et qui se déclenchent lors du passage de personnel (Sté CARMELEC) ;

- des banderoles (Fig. 15) utilisées pour le balisage des zones d'exclusion mentionnant clairement le risque. 
RADIOPROTECTION ET INTERVENTIONS DE RADIOGRAPHIE INDUSTRIELLE

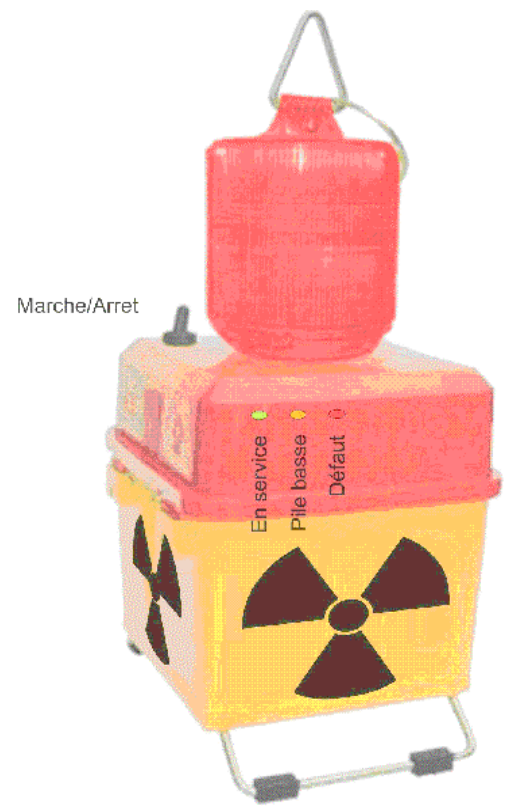

Figure 13 - Balise « sentinelle ».

Gamma gauge.

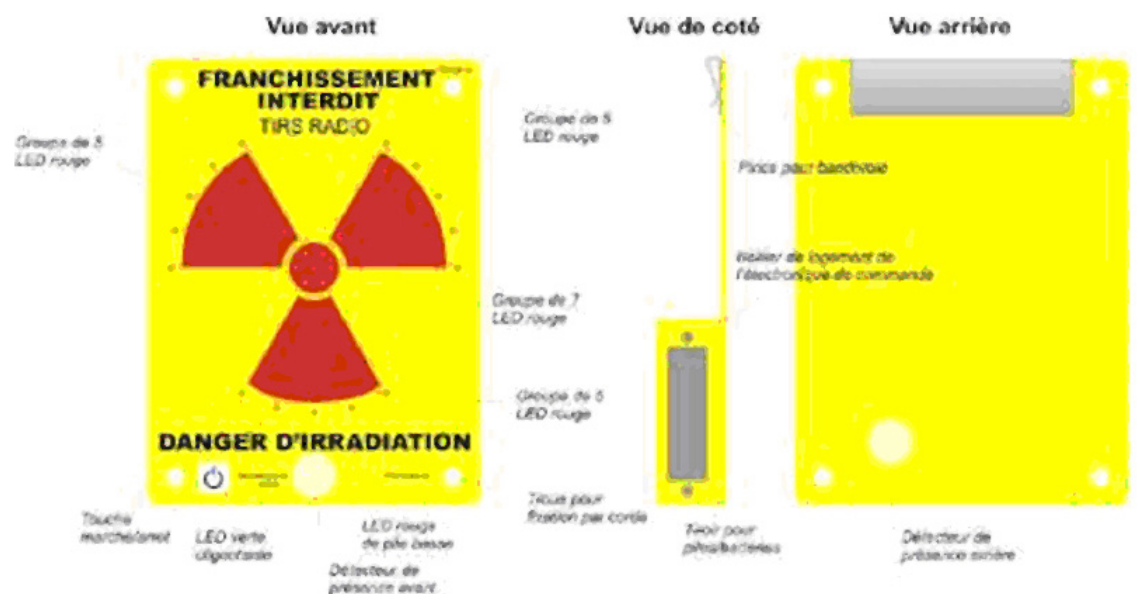

Figure 14 - Délimitation lumineuse de la zone d'interdiction.

Luminous beaconing of the zone of prohibition.

RADIOPROTECTION - VOL. 41 - No 2 (2006) 


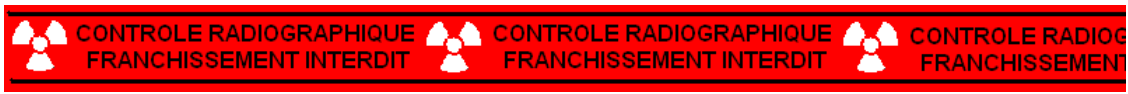

Figure 15 - Balisage standard.

Standard beaconing.

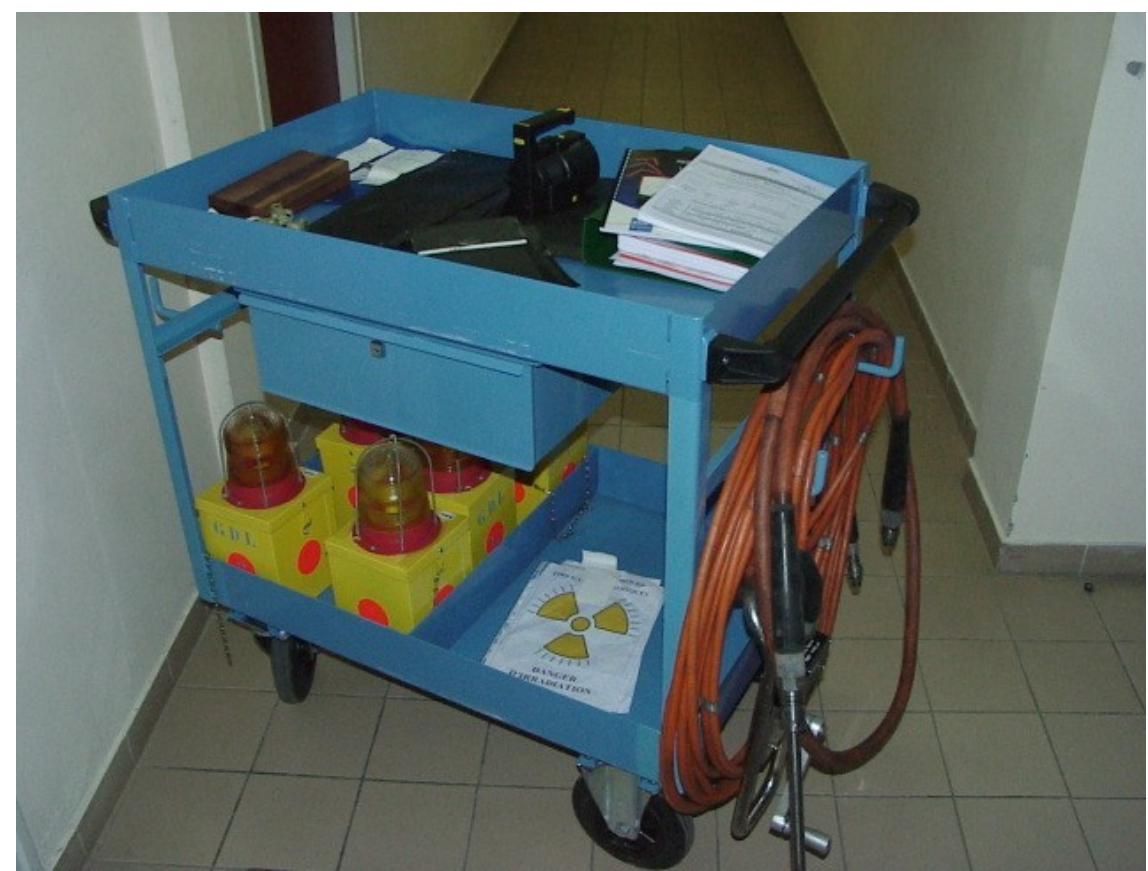

Figure 16 - Chariot de transport de matériel.

Carriage of transport of the material.

\subsection{Contrainte}

Elles imposent la réduction de la contrainte des radiologues et de leur charge de travail : la manutention des gammagraphe, avec les balisages, les cassettes porte film, le radiamètre, ... n'est pas aisée. Des chariots spécialement conçus seront mis à disposition en 2006 (Fig. 16). 


\section{Les bonnes pratiques}

Les « bonnes pratiques » dont certaines ont été prescrites au sein des installations EDF DPN, au titre des « pratiques performantes ». Les « autres bonnes pratiques » sont mises en œuvre par les sites en fonction de leur organisation.

Ces pratiques portent sur:

- les actions liées à la protection de l'environnement des contrôles radiographiques. L'objectif est principalement la protection des personnels non impliqués directement dans la réalisation des contrôles. On peut citer :

- le contrôle du balisage par une personne différente de celle qui le met en place,

- la formalisation du contrôle du balisage sur le plan de qualité,

- désigner une personne en charge de l'ensemble des contrôles de balisage pendant la révision des installations,

- ...

- des actions liées à la protection du personnel réalisant les contrôles radiographiques. Par exemple :

- communiquer aux intervenants un document (type consigne de sécurité) décrivant les gestes à faire en cas d'incident. (Prévenir l'exposition du personnel, informer l'astreinte RP, résoudre le problème, etc.),

- l'équipe du service compétent susceptible d'intervenir d'astreinte est informée du fonctionnement des gammagraphes ;

- des actions visant à réduire la contrainte des radiologues et leur charge de travail :

- faire intervenir lors du même poste de travail les intervenants en zone contrôlée $o u$ hors zone contrôlée, et éviter les transferts de personnel et de matériel ;

- la précision des responsabilités : des documents internes précisent les responsabilités des différents acteurs : une signature sur un permis de contrôle constitue un engagement, et ne doit en aucun cas être considérée comme une formalisation de vérification.

En tant qu'entreprise utilisatrice, au titre du décret 92-158, qui est toujours applicable dans les installations nucléaires de base (INB) le site est responsable de la coordination des travaux et de la fourniture des informations concernant les installations. Si le «client» n'est pas une INB, le décret ne s'applique pas systématiquement.

L'entreprise de radiographie est responsable de la mise en place du balisage, car cette action lui est confiée par le « client». Cependant, cette entreprise n'a pas autorité sur d'autres intervenants sous traitants par exemple. 
Les actions en cours sont actuellement envisagées à EDF la mise en œuvre et la généralisation de pratiques jugées «performantes » issues des sites. On peut citer :

- l'utilisation de plans standardisés, pour permettre d'établir la liste des accès à condamner et pour faciliter l'information des autres intervenants ;

- pour permettre l'implication des services opérationnels du « client », tel que défini et recommandé par l'AIEA (1999), une « charte du radiologue » est mise en œuvre par les donneurs d'ordres. Ces « donneurs d'ordre ne font pas partie des « services compétents en radioprotection», mais des services liés à la maintenance. Au travers de cette charte, l'exploitant s'engage au travers du chargé d'affaire, à fournir un minimum de moyens d'informations aux intervenants. Il met en œuvre les moyens nécessaires à la collecte et à l'utilisation d'un retour d'expérience fiable.

\section{Conclusion}

Les pistes actuelles qui doivent permettre d'assurer une meilleure qualité et une meilleure sécurité dans la mise en œuvre nous semblent être :

- une meilleure planification de ces activités de contrôle au sein des projets. La mise sous assurance de la qualité de la planification et de l'analyse de risque précédant le contrôle radiographique doit permettre d'éviter les interférences entre activités qui sont source d'incident ;

- l'industrialisation de la détermination des zones d'exclusion, en concertation entre l'entreprise intervenante et l'entreprise utilisatrice ;

- l'augmentation du contrôle des activités, dans la mesure où ces contrôles sont associés à une assistance portant notamment sur l'organisation des travaux. L'isolement organisationnel de prestataires travaillant de nuit est un facteur de risque important ;

- le partage d'informations de l'ensemble du personnel présent sur l'installation pendant les activités de contrôle radiographique. Cette information peut prendre plusieurs formes: affichage de plans simples et compréhensibles, messages audio, utilisation des réseaux de communication audio-visuels locaux, etc. ;

- l'amélioration et la formalisation du dialogue entre l'entreprise utilisatrice et l'entreprise intervenante. Ce dialogue doit avoir lieu entre les «personnes compétentes », mais aussi entre les opérationnels ;

- la formation des opérateurs. La responsabilisation des intervenants et leur adaptation aux différents contextes industriels dans le cadre d'une évolution du niveau d'exigence qui doit tendre au «zéro défaut » nécessitent une formation plus approfondie. Ce complément pourrait notamment inclure lors des recyclages périodiques, une analyse des évènements significatifs. 
RADIOPROTECTION ET INTERVENTIONS DE RADIOGRAPHIE INDUSTRIELLE

\section{RÉFÉRENCES}

EAN (2002) 5th European ALARA Workshop "Industrial Radiography, Improvements in radiation protection", http://www.ean.cepn.asso.fr.

Garrigou A., Tannière C., Carballeda G. (2003) Les activités de contrôle non destructif par gammagraphie : "une activité de l'ombre sous les feux de l'actualité", Rapport de recherche/Action, EDF-GDL/LAP-ADS-IUT HSE, Université de Bordeaux 1.

IAEA (1999) Radiation protection and safety in industrial radiography, safety report series no. 13, IAEA, Vienna. 